\title{
Algunas observaciones sobre la tríada ser-vida-intelecto en Plotino y en Proclo
}

\author{
MALENA TONELLI \\ Universidad de Buenos Aires \\ Universidad Nacional de La Plata
}

DOI: $10.36446 /$ rlf2021212

Resumen: En este artículo expondré las interpretaciones que Plotino y Proclo llevan a cabo de la tríada ser, vida e intelecto que Platón presenta en el Sofista. Me concentraré en algunos pasajes de las Enéadas y de la Teología Platónica para examinar los diferentes modos en que cada uno de ellos integra esa tríada en el ámbito intelectual con vistas a analizar la crítica que Proclo emprende del sistema plotiniano a propósito de la conformación del primer grado de generación.

Palabras clave: Sofista, segunda hipóstasis, tradición platónica.

Some Observations on the Triad BeingLife-Intellect in Plotinus and in Proclus

Abstract: In this paper I will study the different interpretations that Plotinus and Proclus carry out of 
the triad being-life-intellect presented by Plato in the Sophist. I will concentrate in some passages of the Enneads and the Platonic Theology to examine the different ways in which each of them integrates this triad in the intellectual sphere in order to analyze, in the last part of the paper, the criticism that Proclus undertakes of the Plotinian system regarding the conformation of the first generation degree.

Key-words: Sophist, second hypostasis, platonic tradition.

- s una opinión generalizada que Plotino y Proclo ocupan un lugar

Cdestacado en la filosofía de la Antigüedad Tardía. Su influencia en la filosofia posterior se encuentra acreditada por numerosos estudios contemporáneos. Recientemente, una considerable cantidad de volúmenes colectivos ha examinado el modo en que estas figuras del llamado neoplatonismo han impactado en las reflexiones de filósofos posteriores, tanto de la Edad Media o del Renacimiento como en filósofos modernos o contemporáneos. ${ }^{1}$

Como se sabe, la continuidad doctrinal entre Plotino y Proclo merece un análisis cuidadoso, puesto que si bien ambos presentan criterios teóricos que sirven para delimitar líneas comunes en muchos aspectos de su pensamiento, la lectura procleana de las Enéadas es, en general, polémica.

Respecto de Plotino, es habitual que se lo ubique como el fundador del llamado "neoplatonismo", especialmente entre los estudiosos de la segunda mitad del s. XX. ${ }^{2}$ De acuerdo con esto, podemos afirmar que la filosofia de Plotino no solo "inicia" una corriente, sino que también sintetiza, profundiza y, por tanto, reelabora las precedentes, pues, como afirman Santa Cruz y Crespo (2007:VII-XI), condensa los principales problemas sobre los que el platonismo discutió por más de seis siglos. En este sentido, Proclo recorre un camino similar. En las últimas décadas, los especialistas lo han ubicado en un lugar destacado dentro de los filósofos de la Antigüedad Tardía. Esto responde a varios motivos: por una parte, su indiscutible influencia en la filosofia posterior, especialmente a partir de las traducciones latinas de su

\footnotetext{
${ }^{1}$ Véanse, por ejemplo, Baine Harris 2002 y Gersh 2014 y 2019.

${ }^{2}$ La valorización de la filosofía plotiniana fue aumentando desde la segunda década del siglo XX en adelante. Cabe destacar el trabajo fundacional de Dodds 1928 a partir del cual se abrió una nueva perspectiva no solamente de la gravitación de Plotino en la historia de la filosofía sino también del interés teórico que las Enéadas suscitan. Respecto de la visión de Plotino como el iniciador de la corriente neoplatónica, véase, por ejemplo, Gatti 1996.
} 
obra; por otra, en tanto fuente de numerosos pensadores antiguos de los que conservamos fragmentos y testimonios gracias a su obra. Actualmente, hay un acuerdo generalizado en que resulta clave comprender el pensamiento del autor en cuya obra se insertan textos que no conservamos en su totalidad. Generalmente, aquellas referencias se encuentran insertadas en un contexto de discusión por lo que el sentido de las citas debe ser evaluado en función del contexto. Por último, la filosofia procleana ha suscitado un interés por sí misma. Su compleja interpretación de lo real en clave henológica, que combina perspectivas científicas, mistéricas, poéticas y exegéticas, lo sitúa en un lugar central pues Proclo sintetiza su atmósfera intelectual y se nutre de sus contemporáneos y predecesores. Ciertamente, hay algunos puntos clave que él mantiene de la, ya desarrollada a su altura, "tradición platónica", sin embargo, como he señalado, en sus escritos hallamos numerosas críticas a la doctrina plotiniana.

En este sentido, como sostiene Radek Chlup (2012: 16), no es sencillo agrupar a pensadores en algunos casos divergentes en una escuela unificada ni establecer una jerarquía de importancia o lugares destacados. ${ }^{3}$ De hecho, no siempre determinado pensador disfrutó de la misma apreciación en diferentes ámbitos intelectuales. En este sentido, Harold Tarrant (2017: 30 y 37) observa que la preeminencia que actualmente se le atribuye a la figura de Plotino ${ }^{4}$ no se debe a que él mismo "hubiera reclamado tal papel" sino que fue su discípulo directo, Porfirio, y acaso el indirecto, Jámblico, ${ }^{5}$ "quienes pusieron al platonismo en el camino hacia Proclo" (Tarrant 2017: 30 y 37). Este autor destaca la tendencia de los últimos años a atender a las contribuciones de Plotino, Porfirio, Jámblico, Siriano y Proclo como un sistema de influencias a partir, de hecho, de los propios testimonios de la obra procleana, pero destaca que el platonismo que Plotino promovió no era en la época de Proclo la "corriente principal". Esto se hace manifiesto si nos concentramos en los numerosos aspectos en los que su doctrina se distancia de la de Plotino.

Ahora bien, en cuanto al sistema de influencias que permite clasificar a diferentes pensadores (aún con una perspectiva crítica) como miembros de una misma tradición -el platonismo de la Antigüedad Tardía-, Chlup (2012: 16) propone una clasificación "geográfica" para designar la diver-

\footnotetext{
${ }^{3}$ Véase, además, Athanassiadi 2006.

${ }^{4}$ Véase, por ejemplo, Gersh 2019: 1-15.

${ }^{5}$ Sobre la figura de Jámblico puede consultarse el importante trabajo de Shaw 1995 o el de Taormina 1999. Recientemente, en nuestra lengua, contamos con la gran contribución de José Molina Ayala 2012.
} 
gencia teórica entre Plotino y Porfirio, de un lado, y Jámblico y sus sucesores, de otro. Mientras que los dos primeros, sostiene este autor, influyeron especialmente en el Occidente latino, pagano o cristiano, no fue tan profundo su impacto en el Este. Fue Jámblico quien "le dio al neoplatonismo una nueva dirección" y Chlup señala que, puesto que Proclo lo valoró mucho más que a Plotino, puede ser inscrito en lo que él llama "tradición jambliqueana oriental" y, en consecuencia, lo clasifica como miembro de un neoplatonismo oriental, "en oposición al neoplatonismo del Occidente latino inspirado en Plotino". 6

No es mi intención defender o cuestionar esta clasificación entre tipos de neoplatonismo, sino tomarla como punto de partida para el análisis que aquí presento. Ciertamente, Plotino y Proclo comparten la visión dinámica de la causalidad, incluyendo la subdivisión en el ámbito inteligible a partir de lo Uno, y los movimientos de procesión y retorno como ejes centrales de la actividad productiva. Asimismo, cada uno a su modo tiene a la filosofía de Platón como fuente última de la sabiduría que se proponen sintetizar y desplegar en función de otras corrientes con las que dialogan o polemizan.

En este marco teórico común, puede afirmarse que Proclo explicita su debate con Plotino en diferentes aspectos; sin embargo, solo me detendré en uno de ellos. Como Plotino, Proclo ha planteado un universo continuo desde un Uno que todo lo excede, pero multiplicó los eslabones en la cadena de intermediaciones aumentando el número de realidades incorpóreas. De este modo, se distancian las causas de los efectos y se complejizan los caminos de ascenso y descenso en un sistema sumamente sofisticado. Por mi parte, en línea con la interpretación más generalizada, entiendo que la necesidad de explicitar tales subdivisiones surge, para Proclo, de la deficiencia explicativa que el sistema hipostático plotiniano presenta a la hora de establecer el primer despliegue desde lo Uno. En efecto, uno de los planteos plotinianos que Proclo profundiza gira en torno de la constitución del ser como primer generado en una línea de continuidad con su generador que garantice, a su vez, la unicidad de todo lo real. De este modo, podría eludirse el problema de la separación que anula la eficacia causal y, por tanto, hace fracasar el sistema explicativo.

En este trabajo, examinaré este aspecto de la crítica procleana para intentar establecer cuál es el fin de esta distancia teórica.Tomaré las interpretaciones que cada uno de estos filósofos llevan a cabo de un pasaje del Sofista,

\footnotetext{
${ }^{6}$ Sobre la influencia de Plotino en autores tanto paganos como cristianos, de lengua griega o latina, véase Emilsson 2017: 373-380.
} 
en donde Platón plantea la tríada ser-vida-intelecto, ya que entiendo que es un buen ejemplo de este sistema de influencias, pues la lectura de la tríada en aquel diálogo por parte de Proclo se nutre tanto de la continuidad de doctrinas jambliqueanas ${ }^{7}$ cuanto de las implicancias teóricas de la de Plotino. ${ }^{8}$ Para ello, expondré brevemente el pasaje en cuestión del Sofista platónico; luego me valdré de algunos pasajes de las Enéadas para mostrar el modo en que Plotino inserta la tríada ser-vida-intelecto en su sistema; finalmente, si bien la tríada es expuesta y analizada en varias obras de Proclo, como en los Elementos de Teología o en el Comentario al Timeo, circunscribiré mi análisis de su interpretación a algunos tramos del Libro III de su Teología Platónica. ${ }^{9}$

\section{Ser, vida e intelecto en el Sofista de Platón}

De los pasajes clave del Sofista que tanto Plotino como Proclo interpretan en estos aspectos, tomaré solamente aquellos que se insertan en el contexto en el que Platón, en boca del Extranjero de Elea, emprende una crítica a los llamados "amigos de las formas" en función de su concepción estática de lo que es. En su distinción entre lo que es y lo que deviene, ellos habrían suscripto el movimiento a lo segundo. En contraste, el Extranjero, mediante las nociones de acción y pasión, sostiene que no es adecuado separar al ser del movimiento. En 248c1, el Extranjero afirma a propósito de los "amigos de las formas":

EXTR. - No concordarán con nosotros en lo que acabamos de decir a los hijos de la tierra sobre la ousía. ${ }^{10}$

TEET. - ¿Por qué?

\footnotetext{
${ }^{7}$ Véase, por ejemplo, Dodds 1963: XXII y 220-221. Para una caracterización sintética de la distancia de Jámblico respecto de la concepción triádica general de lo real en Plotino, véase Bréhier 1948: 475-476.

${ }^{8}$ Véase Dodds 1963: 252-253.

${ }^{9}$ La edición del texto del Sofista aquí utilizada es la de Burnet 1905. Las citas siguen la traducción de Cordero 2000 con algunas modificaciones. Para las Enéadas, sigo la editio minor de Henry y Schwyzer 1964-1982 y las traducciones de Igal 2000 (levemente modificadas) y de Santa Cruz y Crespo 2007 alternativamente. Consignaré en cada caso la traducción utilizada. Las citas y referencias a la Teología Platónica se hacen de acuerdo con la edición de Saffrey y Westerink 2003 y la traducción de Nieva 2016.

${ }^{10}$ En todo este pasaje, Platón se vale del vocabulario de la ousía para referirse al ámbito del ser y al objeto de conocimiento.
} 
EXTR. - ¿Establecimos una definición (hóron) ${ }^{11}$ adecuada de las cosas cuando dijimos que en todo está presente la potencia de padecer (páskhein) o de hacer (drân), incluso en mínima medida?

TEET. - Sí.

EXTR. - A ello responden lo siguiente: el devenir (genései) participa de la potencia de padecer (páskhein) y de actuar (poieîn); pero -dicen- no corresponde a la ousía la potencia de ninguna de estas dos cosas (Sof. 248c1-7).

Para los amigos de las formas, la potencia queda del lado del devenir, no del ser. El lector podría esperar que el Extranjero mostrara que también debe haber potencia en el ámbito de lo que es, sin embargo, no retoma inmediatamente esa noción sino que se detiene en las nociones de "actuar" y "padecer" para introducir las de movimiento y reposo y aplicarlas, a ambas, a los dos términos: devenir y ser.

TEET. - ¿Acaso dicen algo consistente?

EXTR. - Algo sobre lo cual debe decirse que les rogamos que nos informen con más claridad: si están de acuerdo en que el alma conoce (gignóskein) y en que la ousía es conocida (gignóskesthai).

TEET. - Al menos, lo dicen.

EXTR. - ¿Y qué? «¿Dicen que conocer y ser conocido son una acción (poíema), o una afección (páthos), o ambas cosas? ¿ O bien uno es afección, y el otro es lo otro? ¿O ninguno de los dos tiene parte en ninguna de las dos en absoluto?». Es evidente que ninguno de los dos en ninguna de las dos, pues dirían lo contrario de antes. ${ }^{12}$

TEET. - Comprendo.

EXTR. - Pues dirían esto: si conocer (gignóskein) es hacer algo (poieîn ti), ocurrirá necesariamente que lo conocido (gignoskómenon) padece (páskhein). La ousía, que es conocida por el conocimiento en virtud de la razón (katà tòn lógon), al ser conocida experimentará movimiento (kineîsthai) en virtud del padecer, cuando en realidad afirmamos que eso no ocurre en lo estable. TEET. - Es correcto (Sof. 248c8-e5).

Este pasaje ha generado numerosas controversias que no podré ana-

11 Cornford 1935: 240 evita traducir hóros como "definición" y prefiere "a sufficient mark". Respecto de la controversia que la asociación entre ser y potencia ha suscitado, véase Fronterotta 2008.

${ }^{12}$ Sigo la elección de Cordero 2000: 408, n. 196 de no terminar el parlamento de Sócrates en el punto anterior. 
lizar aquí en profundidad. De todos, solo mencionaré un problema que podría tener relación con la interpretación que los platónicos posteriores ofrecieron. Cornford entiende que las tres posibilidades que el Extranjero plantea en este último pasaje resultan en los tres modos posibles de entender el conocimiento: puede ser o bien una acción o una pasión, puede ser ambas simultáneamente o ninguna. Para él, el siguiente parlamento corresponde a lo que los "amigos de las formas" responderían: si el conocimiento implicara una acción, el ser conocido sería afectado por ella por lo que se movería. Por tanto -responderían- el objeto de conocimiento no sería admitido en el ámbito de lo que es puesto que allí no puede haber movimiento. Cornford (1935: 240, n. 2 y 3) explica que, en este pasaje, Platón nos muestra que los amigos de las formas rechazan que el conocimiento sea una acción porque entraría en contradicción con el modo estático en el que comprenden al ser. Esta interpretación fue ampliamente discutida, especialmente por quienes entienden que Platón aquí está mostrando lo contrario: que él mismo defiende que el conocimiento es, efectivamente, una acción y que, por tanto, no debe excluirse al movimiento del ámbito del ser. ${ }^{13}$ Es evidente que esta perspectiva se asocia inmediatamente a la plotiniana o procleana, pues para ellos el conocimiento es actividad ${ }^{14} \mathrm{y}$ coincide en el ámbito intelectual con el ser ya que entienden que no solamente se trata de una asociación aristotélica sino que esto es lo que Platón sostuvo en estos pasajes centrales del Sofista. En este sentido, me interesa destacar que, según esta lectura, las nociones de actuar y padecer relacionadas con la de conocimiento parecen dar lugar a la introducción del movimiento como una posible característica de la ousía, el objeto de conocimiento. Si así fuera, el argumento podría sintetizarse del siguiente modo: si lo real no actúa ni padece y el conocimiento es acción sobre un objeto que padece esa acción, tanto el conocimiento como su objeto deberían excluirse del ámbito de lo real. Entiendo que es por esta consecuencia inaceptable que Teeteto encuentre, en el siguiente parlamento, que esto es decir algo terrible. Allí, el Extranjero afirma la necesidad, que parece desprenderse de lo anterior, de que el movimiento sea incluido en lo que "es totalmente":

\footnotetext{
${ }^{13}$ Véase Gerson 2006: 292-294 y Perl 2014. Para una síntesis de las posibles interpretaciones de estos pasajes, véase Fronterotta 2007: 378, n. 196.

${ }^{14}$ Plotino, a diferencia de Aristóteles, sostiene que enérgeia y kínesis pueden entenderse como intercambiables y ambos, a su vez, asociarse con las nociones de poiein, tal como leemos en la crítica a las categorías aristotélicas en la Enéada VI 1 (26), especialmente los capítulos 15 a 22. Más adelante, volveré sobre este asunto.
} 
EXTR. - ¡Y qué, por Zeus!, ¿nos dejaremos convencer con tanta facilidad de que el movimiento (kínesin), la vida, el alma y el pensamiento (phrónesin) no están presentes (pareînai) en lo que es totalmente (tô pantelôs ónti), ${ }^{15}$ y que esto no vive, ni piensa (phroneîn), sino que, solemne y majestuoso, carente de intelecto (noûnoukékhon), es sin movimiento (akineton) y estático (estós)?

TEET. - Aceptaríamos en ese caso una teoría terrible, Extranjero (Sof. 248e6-249a3).

En estas líneas se descarta que haya que excluir al movimiento, a la vida, al pensamiento de lo que es. Ahora bien, la fórmula tò pantelôs ón resulta tan sugerente como controvertida. Me limitaré, con Cordero (2000: 410, n. 201), a mencionar una de las perspectivas interpretativas más difundidas, según la cual la fórmula refiere a lo que es en general, distinguido de la ousía que designa el objeto afectado por la acción del conocimiento. Si esto es así, el tò pantelôs ón, este ser en general, incluye -además de al movimiento, a la vida, al pensamiento, etcétera- a la ousía que se encuentra en reposo para que, en efecto, dé lugar al conocimiento entendido como el movimiento propio del conocer.

De este modo, quienes defienden esta lectura complementan el análisis con los pasajes siguientes del diálogo en donde el Extranjero explica que no se puede excluir al reposo de entre las cosas que son puesto que el objeto de conocimiento, para ser tal, debe ser estable. Es decir, si la realidad fuera absolutamente mutable, no habría conocimiento. Por tanto, la realidad, tò pantelôs ón, comprende tanto el movimiento cuanto el reposo. En consecuencia, si en el ámbito del ser hay tanto movimiento cuanto reposo, ${ }^{16}$ la caracterización de lo real como inmutable -propia de los amigos de las formas- se encuentra seriamente cuestionada.

La lectura que platónicos posteriores han llevado a cabo de estos pasajes claramente ubica en un mismo ámbito de lo real a la ousía, a la vida, a la phrónesis, al noûs (como facultad del alma), al movimiento y al reposo. En líneas generales, puede decirse que llevan a cabo algunas asociaciones al interior de este conjunto de nociones, asimilando la phrónesis con el noûs (entendido, ya, como una instancia anterior al alma), a la vida con el mo-

${ }^{15}$ Para una referencia a las múltiples interpretaciones que esta fórmula ha suscitado, véase Perl 2014 quien defiende la lectura plotiniana de este pasaje.

${ }^{16}$ Si bien esta lectura es la más difundida, existen interpretaciones alternativas como la de Perl 2014 para quien el movimiento y el reposo no son componentes del tò pantelôs ón, sino que se trata de características propias de lo real en consonancia con la cosmovisión plotiniana del intelecto y el ser. 
vimiento y a la ousía con el objeto de pensamiento que se encuentra en reposo. Así, ser, vida e intelecto constituyen los componentes de lo que es, del ámbito intelectual. En este sentido, como explica Pierre Hadot (1957), la asociación entre ser, vida y pensamiento ha impactado en todo el platonismo y, sostiene este autor, en el caso de Plotino, puede constatarse en la importancia que le atribuye a las nociones de movimiento y de alteridad en su concepción de lo que es. El ser que se identifica con la inteligencia implica el movimiento de la intelección, y tal movimiento, a su vez, implica que ser e inteligencia sean diferentes. Este autor detecta varias fuentes en las que Plotino se basa para su concepción triádica de ser-vida-intelecto. De Platón, el pasaje del Sofista 248c-249a que combina con el del Timeo 39e; de Aristóteles, su caracterización del motor inmóvil como nóesis-noéseos del libro lambda de la Metafísica; y, de los estoicos, su concepción de movimiento. ${ }^{17}$

En lo que sigue, intentaré mostrar el modo en que, sobre la base de una lectura afín de este tramo del Sofista de Platón, Plotino y Proclo construyen sus explicaciones de lo real. Ambos muestran, en función de sus propios argumentos, la razón por la cual en el Sofista Platón dice lo que ellos leen que dice. Ciertamente, difieren en aspectos clave que me propongo poner de manifiesto pues, como he adelantado, Proclo se distancia de Plotino en un diálogo polémico aunque, según entiendo, con vistas a un mismo fin.

\section{Ser, vida e intelecto en la segunda hipóstasis de Plotino}

R ecordemos que, en las Enéadas, Plotino destaca la corresponrelación entre objeto de conocimiento (ser) y sujeto (inteligencia) en la segunda hipóstasis, incluyendo en ella, como advierte Reale (1989), distinciones conceptuales y definitorias en términos de la tríada ser-vida-pensamiento, con el fin de explicar el paso desde una unidad absoluta a la primera multiplicidad (la de la segunda hipóstasis). Al respecto, en su clásico trabajo de 1957 mencionado más arriba, Hadot establece la deuda incuestionable que Plotino tiene con el Sofista (248c) de Platón a propósito de esta distinción entre ser, vida e intelecto en su explicación de la segunda hipóstasis, no solamente en cuanto a su estructura interna sino también en cuanto a su génesis.

${ }^{17}$ Véase Hadot 1957: 113 y ss.; 117 y ss.; 135 y ss. 
La relación entre ser y vida, según entiendo, se inscribe en lo que se comprende como una concepción dinámica del orden de lo real, pues sin vida, es decir, sin movimiento y alteridad, no es posible explicar la cognoscibilidad del ser, ni la capacidad productiva, ni la posibilidad de articular las causas con sus efectos. Y, fundamentalmente, la asociación entre ser, vida e intelecto es indispensable para comprender el ser de la segunda hipóstasis, en tanto principio determinado y unificado y a su vez múltiple en virtud de su derivación de lo Uno. Sin embargo, estas notas constitutivas de la segunda hipóstasis no implican diferentes escalas ontológicas. Ni el ser causa la vida ni la vida el intelecto, pues se trata de tres aspectos asociados con lo inteligible, la intelección y el inteligir que, para Plotino, se encuentran identificados en una unidad inescindible.

En un tratado complejo, elV3 (49) 5, ofrece una serie de argumentos para mostrar que inteligencia-intelección-inteligible son lo mismo pues si la inteligencia es actividad, al verse a sí misma debe verse como tal: inteligiendo. Lo que se ve, el ser, es la intelección, la actividad de inteligir:

Pero si la intelección (he nóesis) y lo inteligible (tò noetón) son lo mismo -pues lo inteligible es algo en acto (enérgeia tis), ya que no es potencia, ni es ininteligible, ni está separado de la vida, ni a su vez ni la vida y el inteligir (voeîn) son cosas otras traídas de fuera como ocurre con una piedra o con algo sin alma- entonces lo inteligible es también la ousía primera. Si, de hecho, es acto y el acto primero (ei oûn enérgeia kaì he próte enérgeia), y el más bello, será intelección (nóesis) e intelección sustancial (ousiódes nóesis) (En.V3 5 30-37 Trad. Igal modificada).

En este sentido, ser, vida e intelecto conforman la tríada constitutiva de la segunda hipóstasis pues no puede darse uno sin el otro. Cada uno de los elementos de esta tríada es condición y consecuencia de los otros dos, en una relación de simultaneidad y autoimplicancia. Al final del capítulo 5 del tratado V3, Plotino concluye la identidad entre sujeto que intelige, acción o acto de intelección y objeto inteligible para sostener el rasgo más propio de la segunda hipóstasis: una inteligencia que se piensa a sí misma.

En efecto, tampoco este intelecto (noûs) está en potencia ni es diferente de la intelección (nóesis), ya que, de lo contrario, su sustancialidad estaría, de nuevo, en potencia. Si, pues, es acto (enérgeia), si su sustancia es acto, quiere decir que es uno e idéntico al acto. Ahora bien, el ser y el inteligible es uno e idéntico al acto. Luego uno será todo a la vez: intelecto (noûs), intelección (nóesis) e inteligible (tò noetón) (En.V3 5 39-44 - Trad. Igal modificada). 
Como se ve, el hecho de que la intelección esté en acto, no como una característica que pueda poseer o no sino como su propia constitución ontológica, permite afirmar que es lo mismo que la ousía pues ella también está en acto. Como ambos son actos primeros, no se puede negar que ser e intelección sean lo mismo. Ahora bien, la introducción de la vida en este contexto (que completa la tríada que Plotino lee en el Sofista) se entiende como una necesidad teórica: si hay algo en actividad no puede quitársele la vida. Entiendo que esta asociación entre enérgeia y zoé tiene como clave de equivalencia la noción de kínesis. En el Sofista, el movimiento que el alma lleva a cabo cuando conoce da lugar a la introducción de la vida (indisociable con el alma) en el ámbito de lo que es. Plotino entiende que la inteligencia tiene un movimiento que no solamente le es propio sino que es constitutivo de su actividad. En el tratado III 7 (45), Plotino explica en qué consiste la eternidad por lo que debe analizar su segunda hipóstasis:

Y quien mira de cerca esta potencia múltiple, en la medida en que es como un sustrato, la llama ousía; luego la llama movimiento (kínesin) en la medida en que ve a la vida, luego reposo (stásin) en la medida en que es en todos los sentidos del mismo modo, y diferencia e identidad (tháteron dè kaì tautón) en la medida en que estos son una unidad (En. III 73-7 - Trad. Igal modificada).

A partir de una combinación de pasajes del Sofista, aquellos que refieren a los cinco géneros con el de 248c citado más arriba, Plotino relaciona a la vida con el movimiento de la inteligencia tal como en el pasaje anterior la relacionaba con su actividad, con el hecho de que la inteligencia (y lo inteligible) esté en acto. ${ }^{18}$ De hecho, en su crítica a las categorías aristotélicas en la EnéadaVI 1 (primera sección del extenso tratado sobre los géneros del ser que Porfirio dividió en tres), dedica los capítulos 15 a 22 a las categorías de actuar y padecer y argumenta, dicho de modo muy breve, que el padecer debe ser eliminado de las categorías y que poieîn puede ser reemplazado por la enérgeia y por la kínesis. Los argumentos son sumamente complejos, solo quiero destacar aquí que Plotino no tiene dificultad en cuestionar doctrinas aristotélicas combinando elementos que en el Sofista se encontraban expuestos de manera sucesiva. Para él, por tanto, la noción de vida puede asociarse simultáneamente con la enérgeia y con el movimiento puesto que, en contraste con Aristóteles, no se trata de cosas diferentes. ${ }^{19}$ Si esto es así, la

\footnotetext{
${ }^{18}$ En el capítulo 13 del tratado VI 7 (38), Plotino explica cómo concibe este movimiento propio de la actividad inteligente asociado con la vida.

${ }^{19}$ Hay muchísimos estudios sobre la crítica de Plotino a las categorías aristotélicas. Para la
} 
propia actividad intelectiva es un movimiento vital que se dirige a un objeto que es sí misma.

Finalmente, es claro que la tríada ser, vida e intelecto sostiene el carácter uni-múltiple de la segunda hipóstasis pues esta autoimplicancia entre sujeto inteligente, movimiento intelectivo y objeto inteligible conforman una unidad múltiple que se genera, simultáneamente, de la primera hipóstasis, en donde la unidad se da de modo absoluto. En efecto, lo Uno genera lo real, lo que es más uno dentro de todo lo que es, en este despliegue simultáneo en términos triádicos pero que no deja de ser el ser, el máximo nivel ontológico, el "ser en plenitud":

La llamada ousía primera no debe ser, pues, una sombra del ser (eínai), sino que ella posee el ser en plenitud (plêres esti tò eînai). Pero el ser es pleno cuando asume la forma del inteligir (noeîn) y del vivir. Inteligir, vivir y ser (tò noeîn, tò zên, tò eînai) se dan entonces simultáneamente en lo que es (en tôi ónti). Si algo es (ón), también es Intelecto (noûs) y, si es Intelecto (noûs), también es: inteligir (noeîn) y ser se dan conjuntamente (En.V 6, 6 18-23, trad. Santa Cruz y Crespo).

En el primer generado, en el ser en plenitud, no hay componentes diferenciados como ocurre, según la interpretación difundida, ${ }^{20}$ en el tò pantelós ón del Sofista de Platón, sino que cada componente es los otros sin jerarquías ontológicas ni causales. Son muchos los pasajes en los que Plotino insiste en la necesidad simultánea de identidad y alteridad para que el ser sea tal (inspirado en los mégista géne) y esos pasajes frecuentemente aluden a la noción de movimiento o, en algunos casos, de vida, para explicar, según su interpretación de aquel pasaje del Sofista 248c, la imposibilidad de concebir al ser estático si es que es, de hecho, inteligible. Es decir, la asociación entre ser, vida e intelecto es indispensable para comprender al ser en tanto que tal, en tanto principio determinado y unificado en virtud de su derivación de lo Uno. Asimismo, el movimiento, propio de lo que es, posibilita la procesión pues en el propio inteligir se establece la condición de posibilidad para la autoconstitución del alma. De este modo, la tríada no solamente es constitutiva del ser de la segunda hipóstasis sino que es fundamental para su eficacia causal. ${ }^{21}$

síntesis que aquí traigo, véase Natali 1999.

${ }^{20}$ Perl 2014, como he señalado, difiere de la interpretación difundida del Sofista y entiende que el mismo Platón concibió las formas como inteligibles, vivientes y en movimiento.

${ }^{21}$ No puedo profundizar aquí la consecuencia de la integración de la tríada en la eficacia 


\section{Ser, vida e intelecto en el Libro III de la Teología Platónica}

— n lo que sigue, intentaré sintetizar la dinámica triádica que Proclo lo $\mathrm{Uno}^{22}$ en algunos pasajes del Libro III de su Teología Platónica. Para ello, intentaré mostrar cómo caracteriza allí al ámbito intelectual, en el que los miembros de la tríada ser, vida e intelecto funcionan como elementos que, a diferencia de Plotino, se constituyen en una jerarquía ontológica. De este modo, introduce la tríada ser-vida-pensamiento como momentos constitutivos que articulan lo real y que lo atraviesan en tanto equivalen a los componentes de la dinámica causal: permanencia-procesión-retorno. No me detendré en la función causal de lo Uno y de las hénadas puesto que mi interés se concentra en el modo en que esta tríada se articula en el marco de la lectura que Proclo ofrece del Sofista de Platón. ${ }^{23}$

Cabe aclarar que Proclo, como explica Chlup (2012: 19), considera al "Intelecto" en dos aspectos: tanto en el que designa lo que viene de lo Uno, afin a la segunda hipóstasis plotiniana, cuanto en el de aquel que designa el subnivel que funciona como tercero, después de la vida y del ser, al interior del plano intelectual. Por otra parte, como afirma Butler (2008), el ser se entiende, a su vez, en dos sentidos, uno estrecho y uno amplio: "En el sentido amplio, refiere a todo lo que es, excluyendo solo la privación y los dioses porque son superabundantes". En el sentido estrecho, se refiere al subnivel que funciona como primero en tanto es los inteligibles distinguidos del intelecto que los capta y de la vida.

En el Libro III de la Teología Platónica, según Saffrey y Westerink (2003: CII), luego de concentrarse en las hénadas divinas en los primeros seis capítulos, a partir del séptimo, Proclo se ocupa de lo que él llama "los dioses inteligibles". ${ }^{24} Y$ a en el capítulo 6 introduce los órdenes de los seres y los divide en cuatro: en la base se encuentra el ser corpóreo sin potencia que depende de cuatro causas anteriores: el Alma, el Intelecto, la Vida y el Ser. ${ }^{25}$

causal de la segunda hipóstasis sino que me concentro en su intervención en la explicación constitutiva de esa instancia ontológica.

22 De entre los muchos estudios acerca de la cuestión problemática de la causalidad de lo Uno y las hénadas véanse el clásico capítulo de Lloyd 1967, los trabajos de Butler 2005 y 2008 y el reciente de Van Riel 2017, por nombrar solo algunos pocos. En nuestra lengua, contamos con las valiosas contribuciones de José Manuel Redondo, por ejemplo, 2020.

23 Para un análisis de la lectura procleana del Sofista véanse, por ejemplo, Charles-Saget 1991; Steel 1992; Jesús de Garay 2013.

${ }^{24}$ Para un análisis del papel de las hénadas en los primeros seis capítulos véase Meijer 1992.

${ }^{25}$ En este apartado, me valgo de mayúsculas cuando consigno los órdenes de los seres, el 
Se ocupa, asimismo, de establecer las distinciones entre ellos, es decir, de mostrar por qué se trata de mónadas diferentes que no pueden ser reducidas unas a otras. Su relación es de dependencia directa, en algunos casos, indirecta, en otros, hecho que sustenta no solamente la jerarquía sino también la irreductibilidad. De este modo, el Alma no es idéntica al Intelecto puesto que algunas almas no participan de él. El Intelecto no es idéntico a la Vida puesto que de la Vida participan seres que no participan del Intelecto, por ejemplo, las plantas. Mientras que el Intelecto dirige su potencia a todo lo que es capaz de conocer $-y$, de ese modo, los hace participar de la Vida-, es mediante la Vida que todos los demás participan del Ser. En este sentido, la Vida funciona como un intermedio que, por una parte, junto con el Intelecto, vivifica a los vivientes, pero por otra, junto con el Ser, les otorga ser a los seres. Como se ve, la extensión de los participantes de la Vida es mayor que la de los participantes del Intelecto, del mismo modo que la de los participantes del Intelecto es mayor que la de los del Alma. Pero a su vez, el Ser es más universal, puesto que hay seres que no tienen ni vida ni intelecto. Estas cuatro mónadas se organizan de modo que en el Alma están las otras tres según participación; en el Intelecto, las otras dos; en la Vida, el Ser.

Para Proclo, en la esfera intelectual, el Ser está más allá de laVida y del Intelecto, pues no se encuentra ni en movimiento ni en reposo. Se vale de los pasajes del Sofista (250c) en donde Platón muestra que ambos -movimiento y reposo- pueden predicarse de él sin que ninguno de ellos se identifique con el Ser:

En el Sofista separa el ser de todos los géneros universales del movimiento. Porque el ser conforme a su naturaleza, dice, no está en reposo ni en movimiento; y lo que no está en reposo ni en movimiento está más allá de la vida (TP III6 129 6-10. Trad. Nieva).

En los pasajes del capítulo 6 en los que Proclo menciona el Sofista de Platón, remite a la distinción triádica que el Extranjero de Elea expone en el pasaje de 248c y ss. Aclara que en lo que es realmente (tò óntos ón) hay ser (tò ón), vida (he zoé), intelecto (ho noûs) y alma. El argumento que Proclo recoge es que este intelecto primerísimo (prótistos noûs) debe moverse necesariamente y el movimiento le viene de la vida. En este sentido, mantiene la equivalencia entre actividad intelectual y movimiento asociados con la vida que Plotino había argumentado. Sin embargo, la actividad del intelecto

ámbito intelectual, la subdivisión triádica, etcétera, para hacer énfasis en las distinciones ontológicas del esquema de Proclo. 
parece no serle propia per se, sino que es algo que le viene. Por otra parte, ese movimiento implica un reposo que tampoco le es propio sino que le viene del Ser. A su vez, Proclo consigna que el Extranjero afirma que Ser, Vida e Intelecto llegan a ser en el Alma pues ella participa de ellos según su propio carácter: ella admite lo que le viene de modo logoeidés. Por tanto, Ser,Vida e Intelecto son anteriores al Alma y conforman lo que para Proclo será la tríada constitutiva del plano intelectual. La distinción entre la relación entre el Ser,Vida e Intelecto y el Alma no contrasta con la concepción plotiniana, pues, para él el Alma (tercera hipóstasis) es posterior al Intelecto (segunda hipóstasis) y él mismo presenta como notas constitutivas de este último aquella tríada presente en el Sofista, tal como lo he desarrollado en el apartado anterior.

Una vez establecida la relación entre la mónada intelectual y la de Alma, Proclo explicará de qué modo las relaciones monádicas se constituyen en interrelaciones triádicas constitutivas de cada mónada. Para ello, parte de la exégesis del Filebo y revela la primera díada que procede de lo Uno (capítulo 7): la del límite y la ilimitación. Ofrece una serie de características de cada miembro de la díada: el límite es uniforme (henoeidés), de él depende que todo sea unidad. La ilimitación, por su parte, es la potencia que procede hacia lo múltiple. Ya puede constatarse, en este capítulo, la relación que Proclo establece entre las tríadas y aquella de entre ellas que es primordial en tanto garantiza la articulación dinámica de la realidad: la tríada de la permanencia, la procesión y el retorno. En virtud del límite hay permanencia y unidad, en virtud de la ilimitación, procesión y multiplicación.

En el capítulo 9, Proclo introduce al tercer miembro de la tríada: el mixto. Se trata del Ser en sí, lo más inteligible que procede del límite y de la ilimitación y participa de ambos. Se encuentra presente aquí el modo en que Proclo entiende a la potencia en el pasaje de 248c citado más arriba a propósito de la caracterización que el Extranjero hace del ser. Según Proclo, pues, el Ser es una combinación de uniformidad y potencia porque es causa de las procesiones y de la multiplicidad, aunque él mismo, en rigor, no solo no es múltiple ni procede, dado que, como había advertido antes, permanece, sino que tampoco es potencia en sentido estricto. Es condición de posibilidad para la potencia.

En este sentido, este mixto es una ousía inteligible (noeté).Viene a la existencia (hyphístatai) a partir de dios -en tanto primera causa-, del límite en tanto segunda y de la ilimitación, en tanto tercera. Proclo aclara que el mixto es uno y no uno: es uno conforme al límite, no uno conforme a la ilimitación.Y es la mezcla conforme al dios que se encuentra antes de la díada. En consecuencia, es una mónada porque participa de lo Uno, es duoeidés porque procede de la díada y es una tríada porque, según lo que Proclo lee 
en el Filebo 64a, es necesario que todo mixto comprenda la belleza, la verdad y la proporción.

Ahora bien, en el capítulo 11 -que Saffrey y Westerink justamente titulan: "La tríada del Filebo: belleza, verdad y proporción"- Proclo desarrolla esta nota, a su vez, triádica del mixto. Explica que el Ser necesita de la belleza para el orden (táxis), de la verdad para la pureza y de la proporción para la unificación (hénosis) de lo mezclado. Infiere por esto último, por lo proporcionado (tò súmmetron), que el mixto es uno, por lo verdadero (tò alethés) que el mixto es realmente (óntos) y que por lo bello (tò kalón) el mixto es inteligible (noetón). En consecuencia, las notas características del tò prótos ón son estas: noetón, óntos ón, henoeidésteron.

Ahora bien, al igual que la primerísima de las hénadas genera el primer Ser -el primer dios inteligible- al intermediario lo generará una hénada intermedia. Si el mixto de la primera tríada es noetón, algo bello y determinado, podrá funcionar como límite de la segunda tríada. Es cierto que ya no es el límite de la primera, pues aquel está libre de participación mientras que el mixto participa de ambos miembros de la díada. Sin embargo, en lo que a la procesión respecta, este límite (el mixto) es lo más limitado que pueda haber, por más que haya en él un componente de ilimitación. Es que es, justamente, ese componente el que le ha permitido proceder, o, más bien, que algo proceda de él en tanto él es permanencia.

La segunda tríada, por tanto, estará conformada por el límite (lo noetón - el aspecto limitado del mixto de la primera), la ilimitación (el aspecto ilimitado del mixto de la primera) y un nuevo mixto, un segundo ser, un segundo dios: la Vida inteligible. La Vida contiene en sí la mezcla de unidad y existencia, por una parte, y de potencia, que le vienen del mixto, por otra. En el capítulo 13, Proclo explica que al ser segunda, subsiste según lo segundo de la primera: la ilimitación. Cabe destacar que esta segunda tríada es intermedia e intermediaria en un sentido esencial, pues dice Proclo que manifiesta lo oculto y uniforme de la primera (el límite) pero como -en tanto potencia- es la procesión de lo que viene después, ella reúne y mantiene en todas partes la multiplicidad inteligible de la tercera (ilimitación). Este segundo ser, la Vida, también participa de la proporción, de la verdad y de la belleza. Mientras que el primer Ser subsiste conforme a la proporción, gracias a lo cual era permanencia, el segundo subsiste conforme a la verdad, gracias a lo cual es procesión, pues en cada mixto hay una tríada. En suma, el primero de los mixtos, el Ser inteligible, se rige por la proporción y permanece; el segundo, la Vida, se rige por la verdad (allí se concentran lo inteligible y lo intelectivo); y el tercero, lo Intelectivo, se rige por la belleza, pues se mueve hacia ella.

Así como en el capítulo 6 y a propósito del Sofista, Proclo había explicado que el Alma participa del Ser, de la Vida y del Intelecto, conforme a 
su propio carácter, la participación de la Vida en el Ser y del Ser en la díada límite-ilimitación constituirá una dinámica similar: si en la primera tríada, cuyo mixto es noetón, se encuentran todas las cosas de modo inteligible conforme al límite, en la segunda, cuyo mixto es la Vida, todo está, según el modo de la Vida, conforme a la ilimitación (capítulo 13).

Finalmente, si hay hénadas más elevadas que otras, unas presidiendo las mónadas más elevadas, otras las menos, hay que aclarar que la primerísima hénada ha manifestado (exéphenen) el Ser inteligible (la primera tríada); la segunda, laVida inteligible (segunda tríada); la tercera manifiesta toda la multiplicidad inteligible, no como causa, no como potencia, sino en acto (capítulo 14). Si el primer Ser, el mixto, es noetón, el segundo -intermedio- será noetón-noerós, ${ }^{26}$ y el tercero, por tanto, será noerós. ${ }^{27}$ Más aún, Proclo explicita que si la primera tríada permanece, la segunda permanece y procede, la tercera retorna, pues se vuelve a los inteligibles para inteligirlos ya no como posibilidad sino en acto. La segunda, por su parte, es la condición de que se lleve a cabo la intelección, pues guarda en sí la relación entre inteligible y la capacidad de intelección, es la potencia que participa de la ilimitación y genera la multiplicidad sin ser múltiple; y la primera es el Ser en cuanto tal (ón autoón), la causa, el objeto de intelección (capítulo 14).

Es cierto que, para Proclo, ser, vida e intelecto se encuentran en cada una de las tres instancias, cada uno a su modo, según su propia naturaleza. Sin embargo, sostiene un encadenamiento en pasos no simultáneos en términos causales que redunda en la sucesión de los momentos de permanencia, de procesión y de retorno. De este modo, se desprende de un problema que la estructura jerárquica de Plotino no ha zanjado: el de la simultaneidad o no de estos momentos. ${ }^{28}$ Para Proclo, como hemos visto, la permanencia es anterior a la procesión que, a su vez, es anterior al retorno. Ahora bien, la distinción en momentos causales de la mano de la multiplicación de instancias intermedias -tanto entre aquello que para Plotino son hipóstasis, como al

${ }^{26}$ La distinción entre inteligible (noetón) e intelectivo (noerós) parece haber sido introducida por Jámblico. Según Chiaradonna y Lecerf 2019, a partir de Jámblico, noerós se transforma en un término técnico para designar "el orden de los dioses ubicados justo debajo del Inteligible y dotados de funciones demiúrgicas".

${ }^{27}$ El análisis de estas relaciones triádicas a la luz del vínculo intelectual se encuentra en Libro IV de la Teología Platónica. En los primeros tres capítulos, como apuntan Saffrey y Westerink 2003: LXXI trata de los dioses inteligibles intelectivos en general, luego los trata en relación con el Fedro y luego con el Parménides.

${ }^{28}$ Este problema ha sido ampliamente discutido por los especialistas, véanse, por ejemplo, Trouillard 1955: 104-109; Gatti 1982: 158 y ss.; Bussanich 1988: 2-3 y 233-236; Emilsson 2007: 101-103. 
interior de cada una- permite distinguir los sistemas de ambos pensadores en un punto central: la relación entre lo inteligible y lo inteligente.

A este respecto, Jean Pépin (1956), en uno de los trabajos clásicos más importantes sobre la relación entre la inteligencia y lo inteligible, ha señalado que los tres términos de la tríada ser-vida-pensamiento son para Plotino cualidades del nô̂s, caracteres que se encuentran entrelazados de modo tal que no pueden aislarse unos de otros (1956:59). Proclo, por su parte, según este autor, ubica la identidad entre inteligencia e inteligible solo en el nivel intermedio de la tríada, en la Vida, mientras que el primer nivel constituye un inteligible puro sin inteligencia y el tercero, una inteligencia sin objeto. De este modo, Proclo rompe con la identidad que Plotino había establecido (1956: 63). A la luz de algunas proposiciones de los Elementos de Teología, Werner Beierwaltes (1990 [1965]) explica que, si bien Proclo pudo haber concebido la esfera del Noûs como heterogénea, lo que intenta demostrar en la diferencia es justamente la in-diferencia fundamental y esencial de la esfera del Noûs y con ello la identidad dinámica y relativa, no absoluta, del todo (1990: 144-145).

Por mi parte, he intentado exponer el modo en que Plotino insiste en que inteligible e inteligente deben ser simultáneos pues no le otorga preeminencia causal a ninguno de los dos. Pensar y ser son lo mismo en tanto aspectos inseparables y autoimplicantes en su segunda hipóstasis. Es cierto que introduce el movimiento de intelección como nexo entre ambos, pero se trata de un nexo inseparable de aquello que une. Proclo, como se ha indicado, propone un sistema de jerarquías en donde el Ser (inteligible) es anterior y más unificado que la Vida (inteligible-intelectivo) que es anterior y más unificada que el Intelecto (intelectivo) en el camino de procesión a la multiplicidad. En este sentido, la procesión no solo se entiende como el pasaje de una instancia ontológica a la siguiente, del ámbito intelectual al del Alma por ejemplo, sino que se da ya al interior del mismo ámbito intelectual en el que lo inteligible ocupa el lugar inicial. En el último capítulo del Libro III de la Teología Platónica, Proclo señala esta distancia que lo separa de Plotino a propósito de la identidad entre inteligible e inteligencia:

En efecto, si todo intelecto es para sí mismo inteligible, tiene esta particularidad en virtud de los dioses inteligibles, porque desde allí 〈viene〉 la plenitud para todos.Y de ese modo lo inteligible está en parte separado del intelecto, preexiste en sí y por sí, y en parte no está fuera del intelecto. Porque hay un inteligible que está unido al intelecto, inteligible coordinado 〈que procede del intelecto〉 trascendente, inteligible participado 〈que procede del intelecto〉 imparticipable, inteligible que existe en sí 〈porque procede del intelecto〉 preexistente, inteligible multiplicado 〈que procede〉 del intelecto monoforme (TP III28 178 15-22. Trad. Nieva). 
He destacado esta visión crítica que Proclo plantea de la segunda hipóstasis plotiniana. Ambos pensadores toman del Sofista de Platón elementos teóricos que contribuyen a la construcción de sus propios sistemas explicativos de la realidad. A esa nota común, deseo agregar que ambos pretenden establecer una mecánica causal capaz de dar cuenta del pasaje desde una unidad absoluta hacia una multiplicidad mediante una cadena de causas y efectos. Parecería que, para Proclo, sin esta multiplicación de tríadas articuladas por la participación, no es posible explicar la unidad, pues para él funcionan como garantía teórica de la continuidad causal. Según entiendo, la crítica a Plotino opera más bien como una corrección en aquellos aspectos en los que Proclo considera que el sistema plotiniano corre el riesgo de interrumpir tal continuidad y, por tanto, de romper la unidad de lo real. Llama la atención que el riesgo lo corra Plotino, quien defiende la uni-multiplicidad de la segunda hipóstasis en términos de unidad simultánea, mientras que Proclo lo contiene estableciendo la jerarquía ontológica en el intelecto y multiplicando las instancias, las interrelaciones y las gradaciones.

Finalmente, si se tiene en cuenta este análisis del modo en que cada una de estas figuras destacadas de la Antigüedad Tardía han integrado la tríada ser-vida-intelecto del Sofista en algunos de sus textos, es posible sostener la línea de continuidad que muchas veces se entiende interrumpida. Ciertamente, a partir de Jámblico la interpretación del pensamiento platónico ha sido transformada y su influencia en Proclo es innegable. Sin embargo, tal transformación podría entenderse como una profundización al interior de la misma tradición platónica griega y pagana. En este sentido, el sistema de Proclo funcionaría como un despliegue de los anteriores, agregando aquellos elementos teóricos -subdivisiones y clasificaciones entrelazadas- mediante los cuales compone un sistema que continúa aquello que Plotino inició. ${ }^{29}$

\section{BIBLIOGRAFÍA}

Athanassiadi, P. (2006), La Lutte pour l'orthodoxie dans le platonisme tardif: De Numénius à Damascius (Paris: Les Belles Lettres).

Baine Harris, R. (2002) (ed.), Neoplatonism and Contemporary Thought: Part One and Part Two (New York: State University of New York Press).

${ }^{29}$ Agradezco las observaciones del/la evaluador/a anónimo/a por haber contribuido a mejorar este artículo. 
Beierwaltes, W. (1990 [1965]), Proclo: I fondamenti della sua metafisica (Milano: Vita e Pensiero: 137-151).

Burnet, J. (1900-1907), Platonis Opera, 5 vols. (Oxford: Clarendon Press).

Bussanich, J. (1988), The One and its Relation to Intellect in Plotinus (Leiden: Brill).

Butler, E. P. (2005), "Polytheism and Individuality in the Henadic Manifold", Dionysius 23: 83-104.

Butler, E. P. (2008), "The Intelligible Gods in the Platonic Theology of Proclus", Méthexis - International Journal for Ancient Philosophy, 21:131-143.

Charles-Saget, A. (1991), "Lire Proclus, lecteur du Sophiste", en P. Aubenque (dir.) y M. Narcy (ed.), Études sur le Sophiste de Platon (Napoli: Bibliopolis, 475-494).

Chiaradonna, R. y Lecerf, A. (2019), "Iamblichus", The Stanford Encyclopedia of Philosophy (Fall 2019 Edition), ed. E. N. Zalta. URL = <https://plato.stanford.edu/ archives/fall2019/entries/iamblichus/>.

Chlup, R. (2012), Proclus: An Introduction (Cambridge: Cambridge University Press).

Cordero, N. (2000), “El Sofista”, en Platón, Diálogos, vol.V, intr. y trad. (Madrid: Gredos, 321-329).

Cornford, F. M. (1935), Plato's Theory of Knowledge: The Theaetetus and the Sophist, intr. trad y com. (London: Routledge and Kegan Paul).

De Garay, J. (2013), “Difference and Negation: Plato's Sophist in Proclus”, en B. Bossi y T. M. Robinson (eds.), Plato's Sophist Revisited (Berlín-Boston: De Gruyter, 225-245).

D’Hoine, P. y Martijn, M. (2017) (eds.), All from One:A Guide to Proclus (Oxford-New York: Oxford University Press).

Dodds, E. R. (1928), "The Parmenides of Plato and the Origin of the Neoplatonic One", The Classical Quarterly, 22:129-142.

Dodds, E. R. (1963), Proclus. The Elements of Theology. A Revised Text with Translation, Introduction and Commentary (Oxford: Clarendon Press).

Emilsson, E. K. (2007), Plotinus on Intellect (Oxford: Clarendon Press).

Emilsson, E. K. (2017), Plotinus (London: Routledge).

Fronterotta, F. (2007), Platone, Sofista, intr., trad. y notas (Milán: BUR).

Fronterotta, F. (2008), "La notion de dunamis dans le Sophiste de Platon: koinônia entre les formes et methexis du sensible à l'intelligible”, en M. Crubellier, A. Jaulin, D. Lefebvre y P. M. Morel (eds.), Dunamis: Autour de la puissance chez Aristote (Louvain-la-Neuve: Peeters, 187-224).

Gatti, M. L. (1982), Plotino e la metafisica della contemplazione (Milano: CUSL).

Gatti, M. L. (1996), “The Platonic tradition and the foundation of Neoplatonism”, en Gerson, Ll. (1997) The Cambridge Companion to Plotinus (Cambridge: Cambridge University Press: 10-37).

Gersh, S. (2014) (ed.), Interpreting Proclus: From Antiquity to the Renaissance (Cambridge: Cambridge University Press).

Gersh, S. (2019) (ed.), Plotinus' legacy: The Transformation of Platonism from the Renaissance to the Modern Era (Cambridge: Cambridge University Press). 
Gerson, Ll. (2006), "The "Holy Solemnity of Forms and the Platonic Interpretation of Sophist", Ancient Philosophy, 26: 291-304.

Gerson, L1. (2010) (ed.), The Cambridge History of Philosophy in Late Antiquity, 2 vols. (Cambridge: Cambridge University Press).

Hadot, P. (1957), "Être, Vie, Pensée chez Plotin et avant Plotin", en Sources de Plotin: Entretiens sur l'Antiquité Classique, 5, 21-29 août 1957 (Vandoeuvres-Genève: Fondation Hardt, 107-157).

Henry, P. et Schwyzer, H.-R. (1964-82), Plotini Opera, 3 vols. (Oxford: Clarendon Press) (editio minor).

Igal, J. (2000), Plotino, Enéadas III-IV, intr., trad. y notas (Madrid: Gredos) vol. 2.

Igal, J. (2000), Plotino, Enéadas V-VI, intr., trad. y notas (Madrid: Gredos) vol. 3.

Lloyd A. C. (1967), "Athenian and Alexandrian Neoplatonism”, en A. H. Armstrong, (1967), The Cambridge History of Later Greek and Early Medieval Philosophy (Cambridge: Cambridge University Press, 302-326).

Meijer, P. A. (1992), "Participation and Henads and Monads in the Theologica Platonica III.1-6”, en E. P Bos y P. A. Meijer (eds.), On Proclus and His Influence in Medieval Philosophy (Leiden: Brill, 65-87).

Molina Ayala, J. (2012), Racionalidad y religión en la antigüedad tardía: Una introducción a Jámblico y a su tratado "Acerca de los misterios de Egipto" (México: UNAM).

Natali, C. (1999), "La critica di Plotino ai concetti di attualità e movimento in Aristotele", en C. Natali y S. Maso (eds.), Antiaristotelismo (Amsterdam: Hakkert, 211-229).

Nieva, J. M. (2016), Proclo, Teología Platónica, trad., introd. y notas (Buenos Aires: Losada).

Pépin, J. (1956), "Élements pour une histoire de la relation entre l'intelligence et l'intelligible chez Platon et dans le néoplatonism", Revue Philosophique de la France et de l'Étranger, 146: 39-64.

Perl, E. D. (2014), “The Motion of Intellect On the Neoplatonic Reading of Sophist 248e-249d", The International Journal of the Platonic Tradition, 8: 135-160.

Reale, G. (1989), Introduzione a Proclo (Bari: Laterza).

Redondo Ornelas, J. M. (2020), "Henadología y ontología, o los dioses y las formas: la metafisica binaria y erótica de Proclo", Theoría. Revista Del Colegio De Filosofía, 36: 59-87.

Saffrey, H. D. y Westerink, L. G. (1968-1997), Proclus, Théologie platonicienne, Texte etabli et traduit par - 6 vols. (Paris: Les Belles Lettres).

Saffrey, H. D. y Westerink, L. G. (2003 [1978]), Proclus, Théologie platonicienne, Tome III: Livre III. Texte etabli et traduit par —, (Paris: Les BellesLettres).

Santa Cruz, M. I. y Crespo, M. I. (2007), Plotino, Enéadas, Textos esenciales, Estudio preliminar, selección de textos, traducción y notas (Buenos Aires: Colihue).

Shaw, G. (1995), Theurgy and the Soul:The Neoplatonism of Iamblichus (Pennsylvania: State University Press).

Steel, C. (1992), "Le Sophiste comme texte theologique dans l'interpretation de Proclus", 
en E. P. Bos y P.A. Meijer (eds.), On Proclus and His Influence in Medieval Philosophy (Leiden: Brill, 51-64).

Taormina, D. (1999), Jamblique Critique de Plotin et de Porphyre (París:Vrin).

Tarrant, H. (2017), Proclus, Commentary of Plato's Timaeus, vol. 1 (Cambridge: Cambridge University Press).

Trouillard, J. (1955), La purification plotinienne (Paris: Presses Universitaires de France).

Van Riel, G. (2017), "Proclus on the One, the Henads and Principles", in Martijn, M. and D'Hoine (eds.) All From One:A Guide to Proclus (Oxford: Oxford University Press, 73-97).

Recibido: 02-06-2020; aceptado: 01-07-2020 